SLAC-PUB-8104

April 1999

\title{
Spin Structure Functions $g_{1}$ and $g_{2}$ for the Proton and Deuteron *
}

\author{
Gregory S. Mitchell \\ University of Wisconsin-Madison \\ Madison, WI 53706 \\ (Representing the E155 Collaboration) \\ Stanford Linear Accelerator Center, Stanford University, Stanford, CA 94309
}

\begin{abstract}
The experiment E155 at SLAC measured the spin structure functions $g_{1}$ and $g_{2}$ of the proton and deuteron. The experiment used deep inelastic scattering of $48.3 \mathrm{GeV}$ longitudinally polarized electrons incident on polarized solid ${ }^{15} \mathrm{NH}_{3}$ and ${ }^{6} \mathrm{LiD}$ targets. The data taken by three independent spectrometers covered a kinematic range of $0.014<x<0.9$ and $1(\mathrm{GeV} / \mathrm{c})^{2}<Q^{2}<40(\mathrm{GeV} / \mathrm{c})^{2}$. Due to the high luminosity and polarization available at SLAC the data on $g_{1}$ are to date the most precise in this kinematic range. The $x$ and $Q^{2}$ dependence of $g_{1}$ has been studied using NLO PQCD fits, allowing extraction of values for the Bjorken sum rule and quark and gluon spin contributions to the nucleon. Results are presented for $g_{1}$ and $g_{2}$ for the proton and deuteron.
\end{abstract}

Presented at the American Physical Society Meeting of the Division of Particles and Fields Los Angeles, CA

January 5-9, 1999

${ }^{*}$ Work supported by Department of Energy contract DE-AC03-76SF00515 and DE-AC02-76ER00881. 


\title{
Spin Structure Functions $g_{1}$ and $g_{2}$ for the Proton and Deuteron
}

\author{
Gregory S. Mitchell (Representing the E155 Collaboration) \\ University of Wisconsin-Madison, 1150 University Avenue, Madison, WI 53706-1390
}

\begin{abstract}
The experiment E155 at SLAC measured the spin structure functions $g_{1}$ and $g_{2}$ of the proton and deuteron. The experiment used deep inelastic scattering of $48.3 \mathrm{GeV}$ longitudinally polarized electrons incident on polarized solid ${ }^{15} \mathrm{NH}_{3}$ and ${ }^{6} \mathrm{LiD}$ targets. The data taken by three independent spectrometers covered a kinematic range of $0.014<x<0.9$ and $1(\mathrm{GeV} / \mathrm{c})^{2}<Q^{2}<40(\mathrm{GeV} / \mathrm{c})^{2}$. Due to the high luminosity and polarization available at SLAC the data on $g_{1}$ are to date the most precise in this kinematic range. The $x$ and $Q^{2}$ dependence of $g_{1}$ has been studied using NLO PQCD fits, allowing extraction of values for the Bjorken sum rule and quark and gluon spin contributions to the nucleon. Results are presented for $g_{1}$ and $g_{2}$ for the proton and deuteron.
\end{abstract}

\section{INTRODUCTION}

Polarized deep inelastic scattering is a powerful tool for studying the internal spin structure of the nucleon [1]. Early results obtained by experiments at SLAC [2,3] and CERN [4] indicated that quarks contribute very little to the nucleon's spin. This contradicted expectations of the naive quark-parton model, and led to the so-called spin crisis. Additional theoretical and experimental studies followed, resulting in a richer view of the nucleon. Experiments at CERN [5,6], SLAC [7-11], and DESY [12] have provided increasing levels of precision and enabled the determination of polarized parton distribution functions using next-to-leading order perturbative QCD calculations and the (AltarelliParisi or DGLAP) evolution equations [6,10]. The main goal of the E155 experiment at SLAC was, for both the proton and deuteron, to make a precision measurement of $g_{1}$ covering a wide kinematic range.

\section{INCLUSIVE DEEP INELASTIC SCATTERING AND POLARIZED STRUCTURE FUNCTIONS}

To study the internal spin structure of the nucleons, the SLAC experiments have used the inclusive deep inelastic scattering of a polarized electron beam incident on a fixed polarized nucleon target. The kinematics of a scattering event are determined by the incident electron energy $\left(E_{0}\right)$ and the energy $\left(E^{\prime}\right)$ and angle $(\theta)$ of the scattered electron with respect to the incident beam direction. The event kinematics are described by the 4 -momentum transfer squared $Q^{2}=4 E_{0} E^{\prime} \sin ^{2}(\theta / 2)$, and the Bjorken scaling variable $x=\frac{Q^{2}}{2 M \nu}=\frac{Q^{2}}{2 M\left(E_{0}-E^{\prime}\right)}$. The Bjorken scaling variable gives the fraction of the nucleon momentum carried by the struck quark.

In the unpolarized case, the cross-section for inclusive measurements is related to the unpolarized structure functions $F_{1}$ and $F_{2}$. In the polarized case, the difference between cross-sections for anti-aligned versus aligned electron spins (denoted $\uparrow$ ) and nucleon spins (denoted $\Uparrow$ ) is given by

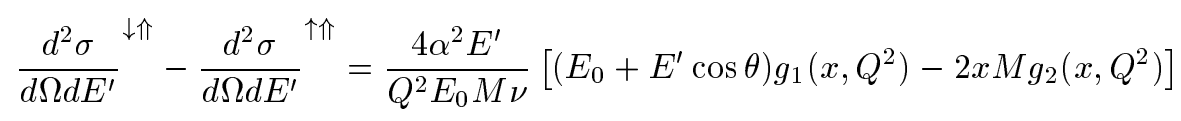

for the case of longitudinal polarization of the target, and by

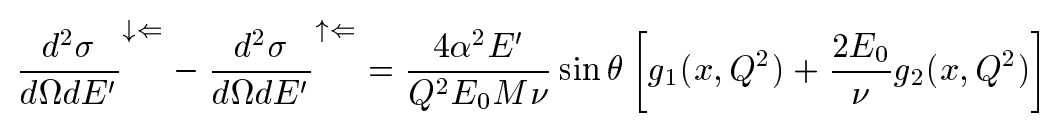

for the case of transverse polarization of the target, where $M$ is the nucleon mass and $\alpha$ is the fine structure constant. The notation $\sigma^{\downarrow \Uparrow}={\frac{d^{2} \sigma}{d \Omega d E^{\prime}}}^{\downarrow \Uparrow}$ (and similarly for other cases) is used below.

Equations (1) and (2) introduce the spin structure functions $g_{1}\left(x, Q^{2}\right)$ and $g_{2}\left(x, Q^{2}\right)$. These functions differ for the various targets (proton $p$, neutron $n$, and deuteron $d$ ), but are related by $g_{1}^{d}=\frac{1}{2}\left(g_{1}^{p}+g_{1}^{n}\right)\left(1-1.5 \omega_{D}\right)$. The factor $\left(1-1.5 \omega_{D}\right)$ corrects for the D-state probability of the deuteron, $\omega_{D}=0.05 \pm 0.01$. This probability is treated as a constant with respect to Bjorken $x$. 
Since in both cases on the left hand side of Eqs. (1) and (2) the cross-sections are nearly equal, to measure $g_{1}$ and $g_{2}$ by measuring the cross-sections and taking the differences would require detailed and accurate knowledge of detector acceptance and efficiency. Instead, the spin structure functions are obtained by measuring asymmetries, where these common factors divide out.

Measuring cross-section asymmetries $A_{\|}$and $A_{\perp}$,

$$
A_{\|}=\frac{\sigma^{\downarrow \Uparrow}-\sigma^{\uparrow \Uparrow}}{\sigma^{\downarrow \Uparrow}+\sigma^{\uparrow \Uparrow}} \quad \text { and } \quad A_{\perp}=\frac{\sigma^{\downarrow \Leftarrow}-\sigma^{\uparrow \Leftarrow}}{\sigma^{\downarrow \Leftarrow}+\sigma^{\uparrow \Leftarrow}}
$$

yields the polarized structure functions via

$$
g_{1}=\frac{F_{1}}{D^{\prime}}\left[A_{\|}+A_{\perp} \tan (\theta / 2)\right] \quad \text { and } \quad g_{2}=\frac{F_{1}}{D^{\prime}} \frac{y}{2 \sin \theta}\left(-A_{\|} \sin \theta+A_{\perp} \frac{E_{0}+E^{\prime} \cos \theta}{E^{\prime}}\right) .
$$

The quantities $g_{1}, g_{2}, F_{1}, A_{\|}$, and $A_{\perp}$ above are all functions of $x$ and $Q^{2}$. The kinematic variables used in the above expressions are determined from $E_{0}$ and either $\left(x, Q^{2}\right)$ or $\left(E^{\prime}, \theta\right)$ as follows: $y=\nu / E_{0}, z=x M / E_{0}, \epsilon=$ $1 /\left[1+2\left(1+\nu^{2} / Q^{2}\right) \tan ^{2}(\theta / 2)\right], \gamma^{2}=4 M^{2} x^{2} / Q^{2}$, and $D^{\prime}=(1-\epsilon)(2-y) /\left[1+\epsilon R\left(x, Q^{2}\right)\right]$. $F_{1}$ was calculated from fits to world data on $F_{2}$ [13] and $R$ [14].

In leading order $g_{1}$ is only sensitive to the net quark polarizations. However, in next-to-leading order (NLO) perturbative QCD (PQCD) $g_{1}$ is also sensitive to the polarized gluon distribution $\Delta G$ through the $Q^{2}$ evolution of the polarized parton distributions. This evolution is governed by the Altarelli-Parisi (or DGLAP) evolution equations [15] and reflects the increasing resolution of deep inelastic scattering with increasing $Q^{2}$. One important consequence of this evolution is to link the quark distributions to the gluon distributions through $\mathrm{g} \rightarrow \mathrm{gq}$ splitting. This makes it possible to indirectly measure the gluon distributions by measuring the quark distributions over a wide range in $Q^{2}$. Given sufficient statistical power over a wide range in $x$ and $Q^{2}$, NLO PQCD analysis of the $Q^{2}$ dependence of $g_{1}$ can be used to constrain the net polarization of the gluon.

Although the naive picture of the $g_{2}$ structure function is unclear, twist-2 calculations from Wandzura and Wilczek [16] provide a relation between $g_{2}$ and $g_{1}$ :

$$
g_{2}^{W W}\left(x, Q^{2}\right)=-g_{1}\left(x, Q^{2}\right)+\int_{x}^{1} g_{1}\left(\zeta, Q^{2}\right) d \zeta / \zeta
$$

More generally, $g_{2}\left(x, Q^{2}\right)$ can be written as $g_{2}\left(x, Q^{2}\right)=g_{2}^{W W}\left(x, Q^{2}\right)-\int_{x}^{1} \frac{\partial}{\partial y}\left(\frac{m}{M} h_{T}\left(y, Q^{2}\right)+\xi\left(y, Q^{2}\right)\right) \frac{d y}{y}$, where the second and third terms are twist-2 and twist-3, respectively.

\section{THE EXPERIMENT}

The experiment E155 ran in early 1997 and collected $~ 180$ million deep inelastic scattering electron events. Keys to the success of E155 included: the high intensity, highly-polarized beam; the use of lithium deuteride as the deuteron target material; and the construction of a third spectrometer.

Longitudinally polarized $48.3 \mathrm{GeV}$ electron beam pulses [17] of up to $400 \mathrm{~ns}$ duration were produced at $120 \mathrm{~Hz}$ by a circularly polarized laser beam illuminating a strained GaAs photocathode. The beam polarization $P_{b}=0.810 \pm 0.020$ was determined using Møller scattering from 20-154 $\mu \mathrm{m}$ thick Fe-Co-V polarized foils [18].

Two polarized nucleon target materials were used in E155: ammonia $\left({ }^{15} \mathrm{NH}_{3}\right)$ as a proton target; and lithium deuteride $\left({ }^{6} \mathrm{Li}^{2} \mathrm{H}\right.$, or $\left.{ }^{6} \mathrm{LiD}\right)$ [19] as a deuteron target. As in E143 [20], polarization of the target materials was obtained using the technique of dynamic nuclear polarization (DNP). In this technique, a combination of microwaves $(\sim 140 \mathrm{GHz})$, low temperature $(1 \mathrm{~K})$, and a high magnetic field $(5 \mathrm{~T})$ polarizes paramagnetic electron centers in the target material, and transfers that polarization to the nucleons. Nuclear magnetic resonance (NMR) measurements were made using coils embedded in the target materials to determine the polarization at regular intervals. These measurements were calibrated to the signal measured at thermal equilibrium near 1.6 K [21]. The polarizations slowly 
decreased over time due to radiation damage, and were restored by periodic annealing at about $80 \mathrm{~K}$ for the $\mathrm{NH}_{3}$ target and $185 \mathrm{~K}$ for the $\mathrm{LiD}$. As compared to deuterated ammonia $\left({ }^{15} \mathrm{ND}_{3}\right)$, lithium deuteride provides a larger ratio of polarized (effective) deuterons to the total number of nucleons as well as higher radiation resistance. The ${ }^{6} \mathrm{Li}$ can, to first order, be treated as a polarized deuteron plus an unpolarized alpha particle, and therefore half of the nucleons in ${ }^{6} \mathrm{LiD}$ are the desired polarizable species. Average polarizations of $\left\langle P_{t}\right\rangle \approx 80 \%$ for the proton target and $\left\langle P_{t}\right\rangle \approx 22 \%$ for the deuteron target were achieved. Overall relative uncertainties on $\mathrm{P}_{t}$ of $6 \%$ (preliminary) and $4 \%$ were obtained for the proton and deuteron respectively.

Scattered electrons were detected in three independent magnetic spectrometers at central angles of $2.75^{\circ}, 5.5^{\circ}$, and $10.5^{\circ}$ with respect to the incident beam, as shown in Fig. 1 . The spectrometers at $2.75^{\circ}$ and $5.5^{\circ}$ were used previously in the experiment E154 [9]. In each spectrometer, electrons were identified by threshold gas Cherenkov counters and a total absorbing lead glass electromagnetic calorimeter. Particle momenta and scattering angles were measured with sets of scintillator hodoscopes. The $10.5^{\circ}$ spectrometer was added for E155 to double the $Q^{2}$ range of the experiment.
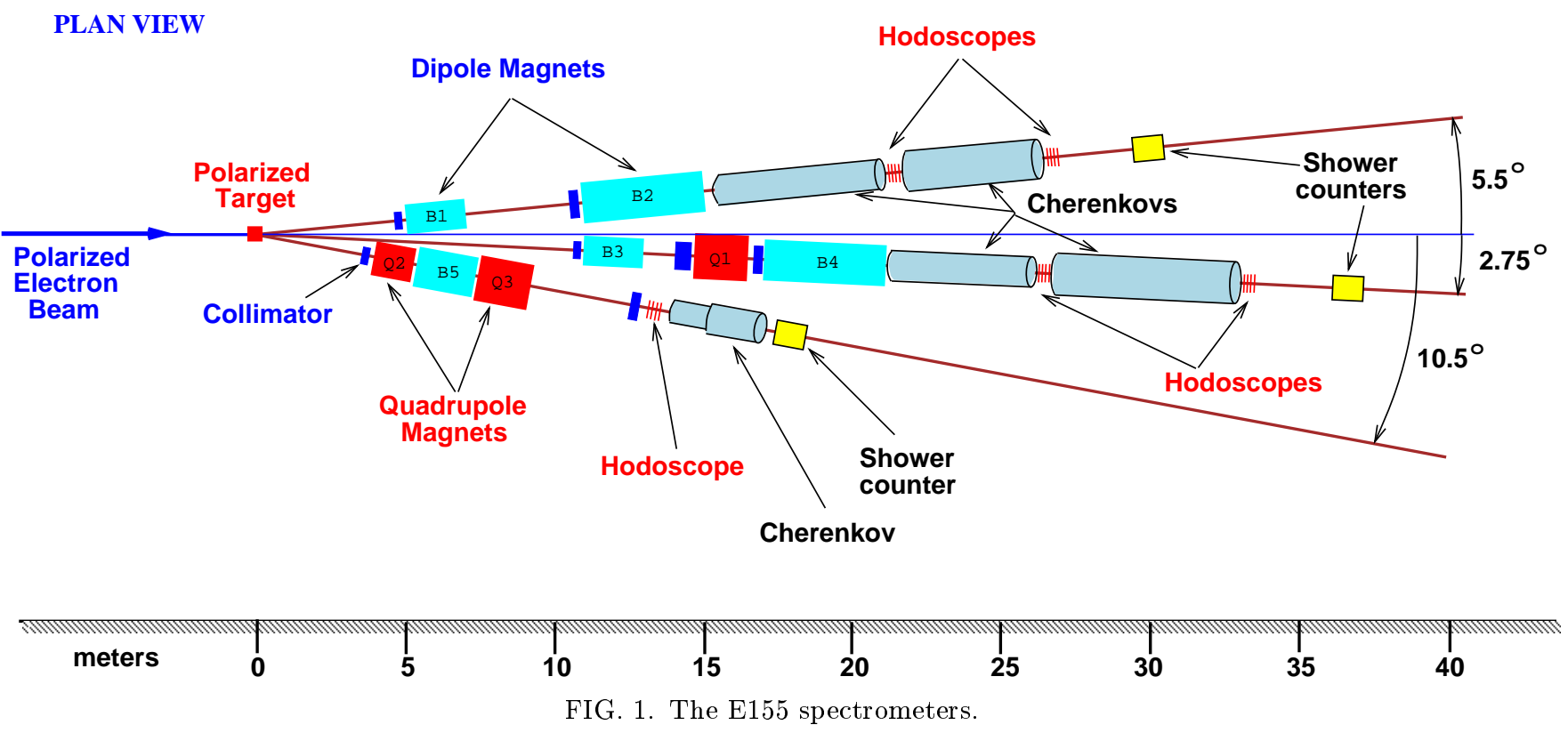

\section{EXPERIMENTAL ASYMMETRIES}

The experimental asymmetries were determined from the numbers of scattered electrons per incident beam charge for negative and positive beam helicity for each target, divided by the beam and target polarizations. The rates were corrected for contributions from charge symmetric background processes, which were measured by reversing the spectrometer polarities. Corrections were also included for mis-identified hadrons, which were typically $2 \%$ or less of the electron candidates. Also, a dilution factor accounted for the fraction of events originating from the polarizable protons or free deuterons, as opposed to the other materials in the target. The average dilution factor for the proton target was 0.15 , for the deuteron target 0.19 .

Nuclear correction factors accounted for the presence of several polarizable nuclear species in the target. For the proton, a small correction was made for the polarization of the nitrogen nuclei. For the deuteron, the measured asymmetry included contributions from the free deuterons and effective deuterons in ${ }^{6} \mathrm{Li}$. The effective deuteron in ${ }^{6} \mathrm{Li}$ has a net polarization of $87 \%$ of the ${ }^{6} \mathrm{Li}$ polarization [22], which leads to an effective dilution factor of $\sim 0.36$ for ${ }^{6} \mathrm{LiD}$, as compared with $\sim 0.22$ for ${ }^{15} \mathrm{ND}_{3}$. The measured deuteron asymmetries also were corrected for small contributions from polarized protons in $\mathrm{Li}^{1} \mathrm{H}$ and ${ }^{7} \mathrm{Li}$.

Both internal [23] and external [24] radiative corrections were obtained using an iterative global fit of all data, including E155. Previous SLAC data were recorrected in a manner consistent with the E155 corrections. 


\section{RESULTS}

\section{A. Longitudinal Structure Function $g_{1}$}

The E155 proton results are plotted as $g_{1}^{p}$ vs. $Q^{2}$ in Fig. 2. There is a remarkable agreement between the many experiments. The world data on $g_{1}^{p}$ exhibit a clear $Q^{2}$ dependence, and the scaling violation is similar in character to that of the unpolarized structure functions. At low $x$, the spin structure function increases with $Q^{2}$, and at high $x$ it decreases with increasing $Q^{2}$.

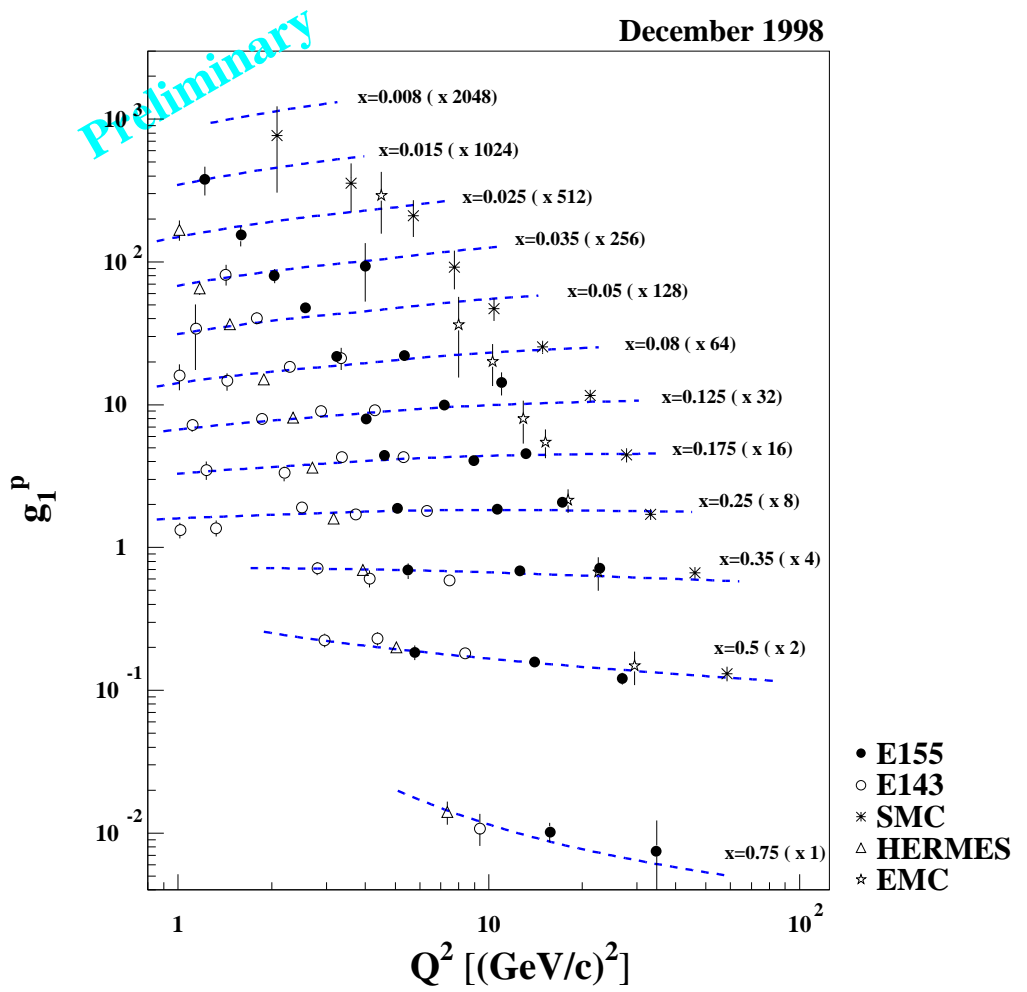

FIG. 2. $g_{1}^{p}$ for E155 and other experiments vs. $Q^{2}$. The dashed curves are from a functional fit to world $g_{1}$ data. The points and curves have been scaled by the factors in parentheses for clarity.

The E155 deuteron results [11] are presented as $g_{1}^{d} / F_{1}^{d}$ vs. $Q^{2}$ in Fig. 3. They are also in good agreement with world data, and the data from the three spectrometers provide both large $Q^{2}$ coverage and good statistical resolution in the mid- $x$ region. There is no significant $Q^{2}$ dependence for $g_{1}^{d} / F_{1}^{d}$, and likewise $g_{1}^{p} / F_{1}^{p}$, indicating that the polarized and unpolarized structure functions evolve similarly.

Evolving the data to a common $Q^{2}$ by use of a functional fit to world $g_{1}$ data, and integrating over the kinematic $x$ range of E155 yields preliminary results for $Q^{2}=5(\mathrm{GeV} / \mathrm{c})^{2}: \int_{0.014}^{0.9} g_{1}{ }^{p} d x=0.132 \pm 0.002 \pm 0.010$ and $\int_{0.014}^{0.9} g_{1}{ }^{d} d x=0.044 \pm 0.003 \pm 0.003$, where the first error is statistical and the second is systematic. The extrapolations for the unmeasured high $x$ region are negligible. However, the contribution from the low $x$ region below 0.014 does not converge for the functional fit, reinforcing the need for additional data at very low $x$. Using the E154 [10] PQCD fit to calculate the contribution for the low $x$ region gives values: $\int_{0}^{1} g_{1}^{p}\left(x, Q^{2}\right) d x=\Gamma_{1}^{p}=0.126 \pm 0.003 \pm 0.010 \pm 0.009$ and $\int_{0}^{1} g_{1}^{d}\left(x, Q^{2}\right) d x=\Gamma_{1}^{d}=0.030 \pm 0.005 \pm 0.004 \pm 0.005$, where again the first error is statistical, the second is systematic, and the third error is a theory error. These values can be combined to obtain a preliminary E155 result for the Bjorken sum rule [25], $\Gamma_{1}^{p-n}=\int_{0}^{1} g_{1}{ }^{p}-g_{1}{ }^{n}=\int_{0}^{1} g_{1}{ }^{p}-\left(g_{1}{ }^{d} \frac{2}{\left(1-1.5 \omega_{D}\right)}-g_{1}{ }^{p}\right)=0.187 \pm 0.012 \pm 0.022 \pm 0.021$. This is consistent with the theoretical prediction at $Q^{2}=5(\mathrm{GeV} / \mathrm{c})^{2}, \Gamma_{1}^{p-n}=0.182 \pm 0.005$, which includes third order QCD corrections [26], using $\alpha_{s}\left(M_{Z}^{2}\right)=0.119 \pm 0.002[27]$. 
Using the published NLO PQCD fit methods of E154 [10], but including final SMC results [5] and preliminary E155 results in the data set, polarized parton distributions were extracted. The eight-parameter fit had a $\chi^{2} /$ d.o.f. of 206/210. From the parton distributions, the following preliminary integral results are obtained at $Q^{2}=5(\mathrm{GeV} / \mathrm{c})^{2}$, in the $\overline{\mathrm{MS}}$ scheme: $\Gamma_{1}^{p}=0.116 \pm 0.005 \pm 0.009, \Gamma_{1}^{d}=0.028 \pm 0.004 \pm 0.007$, and $\Gamma_{1}^{p-n}=0.172 \pm 0.005 \pm 0.008$, where the first errors are statistical and the second systematic, and similarly sized theory errors are ignored. The Bjorken sum rule result is again consistent with the theoretical prediction. Additionally, the fit polarized parton distributions yield the preliminary results: $\Delta G=1.8 \pm 0.6 \pm 1.3$ and $\Delta \Sigma=0.22 \pm 0.04 \pm 0.06$. The data indicate that the gluon contribution to nucleon spin $\Delta G$ is positive, but do not constrain the value well. The data do well constrain the quark spin contribution $\Delta \Sigma$, and it is smaller than predicted naively or by the Ellis-Jaffe sum rule [28].

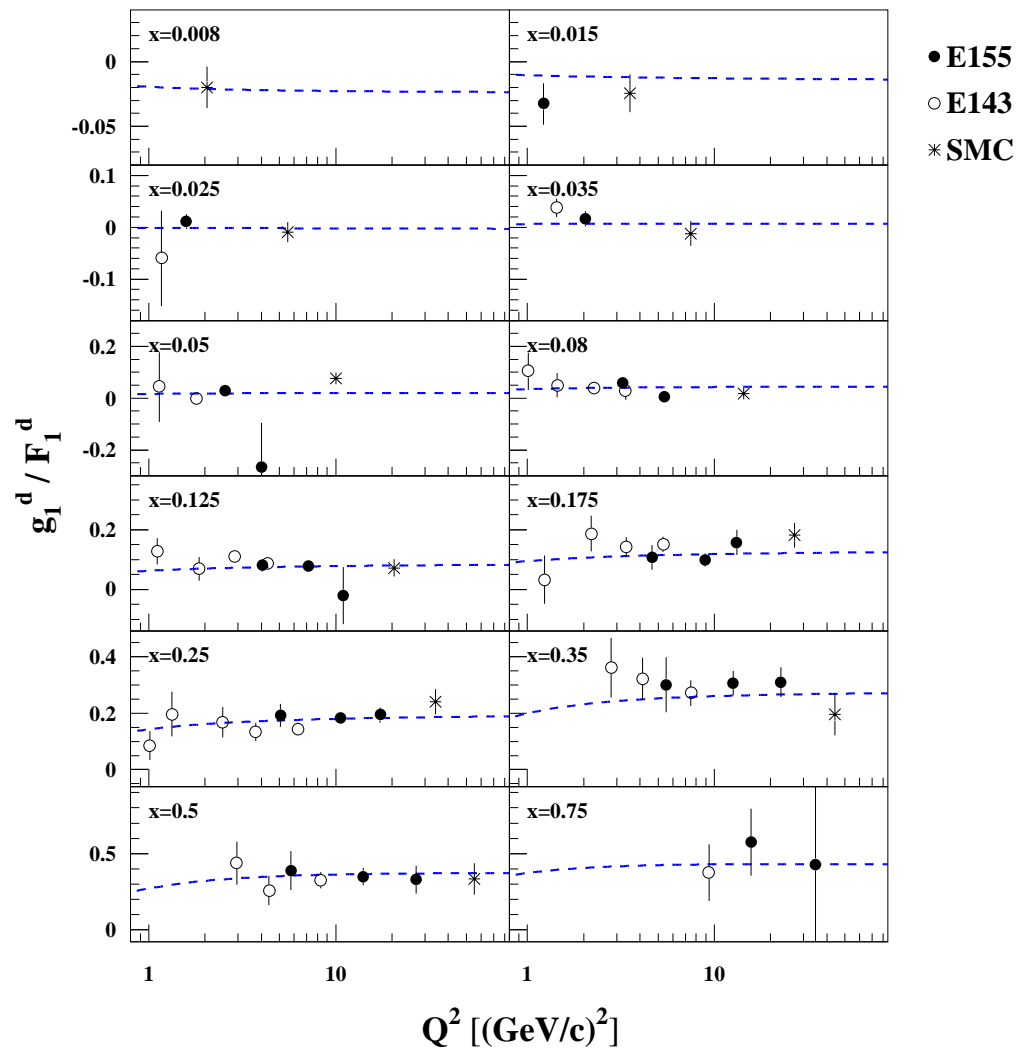

FIG. 3. $g_{1}{ }^{d} / F_{1}{ }^{d}$ for E155 vs. other experiments. The multiple points in each bin for E155 are for the separate spectrometers. The dashed curves are from a functional fit to world data on $g_{1} / F_{1}$.

\section{B. Transverse Structure Function $g_{2}$}

E155 ran for a short period during the 1997 run at $38.8 \mathrm{GeV}$ beam energy with the target polarization transverse to the beam direction. Results for the $g_{2}$ structure function obtained from this data [11] are shown (as $x g_{2}$ ) in Fig 4 . The data from the three spectrometers show no $Q^{2}$ dependence to the virtual photon-nucleon asymmetry $A_{2}$ within uncertainties, so the data from the individual spectrometers have been combined. The data are consistent with $g_{2}^{W W}$, and satisfy the Burkhardt-Cottingham sum rule [29] within errors. However, the current results lack the power to differentiate among $g_{2}^{W W}$, model predictions [30,31], and $g_{2}\left(x, Q^{2}\right)=0$. This will be addressed by the much larger data set planned for the E155 extension run (March-May 1999), which will focus on measurements with the target polarization perpendicular to the beam direction in order to make a high precision measurement of the $g_{2}$ structure function. The data from this run will significantly improve knowledge of $g_{2}$ and will definitively distinguish between $g_{2}=0$ and $g_{2}^{W W}$. 

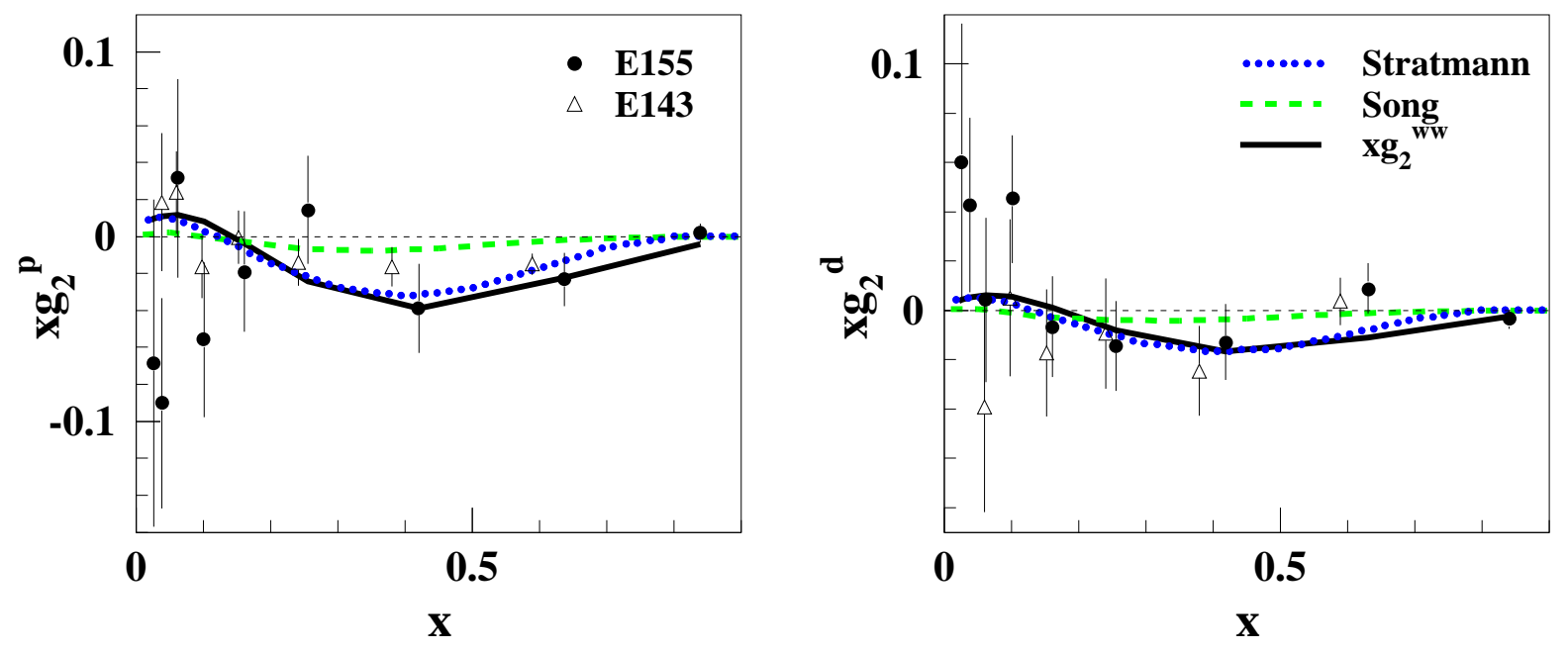

FIG. 4. Plot of $x g_{2}$ for proton and deuteron. The error bars are statistical. The solid line indicates the prediction from Wandzura and Wilczek [16] using a fit to world $g_{1}$ data. Also shown are calculations from Stratmann [30] and Song [31].

[1] R. G. Roberts, The Structure of the Proton, Cambridge Univ. Press (1990).

[2] SLAC E80, M. J. Alguard et al., Phys. Rev. Lett. 37, 1261 (1976).

[3] SLAC E130, G. Baum et al., Phys. Rev. Lett. 51, 1135 (1983).

[4] EMC, J. Ashman et al., Nucl. Phys. B 328, 1 (1989).

[5] SMC, B. Adeva et al., Phys. Rev. D 58, 112001 (1998).

[6] SMC, B. Adeva et al., Phys. Rev. D 58, 112002 (1998).

[7] SLAC E142, P. L. Anthony et al., Phys. Rev. D 54, 6620 (1996).

[8] SLAC E143, K. Abe et al., Phys. Rev. D 58, 112003 (1998).

[9] SLAC E154, K. Abe et al., Phys. Rev. Lett. 79, 26 (1997); Phys. Lett. B 404, 377 (1997).

[10] SLAC E154, K. Abe et al., Phys. Lett. B 405, 180 (1997).

[11] SLAC E155, P. Anthony et al., Report No. SLAC-PUB-7983 (1998); Report No. SLAC-PUB-8041 (1999).

[12] HERMES, A. Airapetian et al., Phys. Lett. B 442, 484 (1998); K. Ackerstaff et al., Phys. Lett. B 404, 383 (1997).

[13] NMC, M. Arneodo et al., Phys. Lett. B 364, 107 (1995).

[14] SLAC E143, K. Abe et al., Report No. SLAC-PUB-7927 (1998).

[15] G. Altarelli and G. Parisi, Nucl. Phys. B 126, 298 (1977); V. N. Gribov and L. N. Lipatov, Sov. J. Nucl. Phys. 15, 438, 675 (1972); Yu. L. Dokshitzer, Sov. Phys. JETP 46, 461 (1977).

[16] S. Wandzura and F. Wilczek, Phys. Lett. B 72, 195 (1977).

[17] R. Alley et al., Nucl. Instrum. Methods Phys. Res., Sect. A 365, 1 (1995); J. E. Clendenin et al., Report No. SLAC-PUB7619 (1997).

[18] H. R. Band, G. Mitchell, R. Prepost, and T. Wright, Nucl. Instrum. Methods Phys. Res., Sect. A 400, 24 (1997).

[19] S. Bültmann et al., Report No. SLAC-PUB-7904 (1998), accepted by Nucl. Instrum. Methods Phys. Res., Sect. A.

[20] D. G. Crabb and D. B. Day, Nucl. Instrum. Methods Phys. Res., Sect. A 356, 9 (1995).

[21] D. G. Crabb and W. Meyer, Annu. Rev. Nucl. Part. Sci. 47, 67 (1997).

[22] O. A. Rondon, Report No. aps1998dec15_002, 1998, submitted to Phys. Rev. C.

[23] T. V. Kukhto and N. M. Shumeiko, Nucl. Phys. B 219, 412 (1983); I. V. Akusevich and N. M. Shumeiko, J. Phys. G 20, 513 (1994).

[24] Y. S. Tsai, Report No. SLAC-PUB-848 (1971); Y. S. Tsai, Rev. Mod. Phys. 46, 815 (1974).

[25] J. D. Bjorken, Phys. Rev. 148, 1467 (1966).

[26] S. A. Larin and J. A. M. Vermaseren, Phys. Lett. B 259, 345 (1991); S. A. Larin, Phys. Lett. B 334, 192 (1994).

[27] Particle Data Group, Eur. Phys. J. C 3, (1998).

[28] J. Ellis and R. Jaffe, Phys. Rev. D 9, 1444 (1974); D 10, 1669 (1974).

[29] H. Burkhardt and W. N. Cottingham, Ann. Phys. 56, 453 (1970).

[30] M. Stratmann, Z. Phys. C 60, 763 (1993).

[31] X. Song, Phys. Rev. D 54, 1955 (1996). 